\title{
La hipertensión pulmonar en el recién nacido con cierre del ductus arterioso en el útero y secuencia de la perfusión arterial reversa en el embarazo gemelar
}

\section{Rocío Villamizar-Durán*, Walter Mosquera, Jaiber Gutiérrez, Juanita Mena y Gerardo Guzmán}

Servicio de Cardiología Pediátrica, Fundación Valle de Lili, Cali, Colombia

Recibido el 29 de abril de 2016; aceptado el 1 de noviembre de 2016

Disponible en Internet el 17 de enero de 2017

\section{PALABRAS CLAVE}

Hipertensión arterial pulmonar;

Ductus arterioso;

Embarazo

\begin{abstract}
Resumen
Introducción: La hipertensión pulmonar del recién nacido secundario con cierre en el útero del ductus arterioso es una complicación frecuente en las pacientes gestantes que usan analgésicos no esteroideos durante el embarazo. Se presenta un caso de secuencia de la perfusión arterial reversa en el embarazo gemelar con evidencia de supervivencia del gemelo acárdico con el flujo sanguíneo del gemelo bomba. El tratamiento es la cirugía con fetocoagulación láser al gemelo acárdico, previniendo complicaciones mayores para el gemelo sano.

Caso clínico: Gemelo con secuencia twin reversed arterial perfusion. A las 22 semanas de gestación se le practicó fotocoagulación con láser al feto acárdico. La madre recibió por dos días acetaminofén y analgésicos no esteroideos para manejo del dolor. Se le realizó ecocardiograma fetal a las 31 semanas de gestación, con evidencia de disfunción diastólica del ventrículo derecho y cierre del ductus arterioso. Se le realizó cesárea de urgencia obteniendo recién nacido que presentó hipertensión pulmonar en el período neonatal, quien con manejo médico tiene resolución de la misma.

Conclusión: La hipertensión pulmonar del recién nacido secundario a cierre del ductus arterioso en el útero por ingesta de medicamentos, es una entidad que pone en riesgo la vida del feto, por lo cual su diagnóstico y tratamiento oportuno es indispensable para mejorar la sobrevida de nuestros pacientes.

(c) 2016 Sociedad Colombiana de Cardiología y Cirugía Cardiovascular. Publicado por Elsevier España, S.L.U. Este es un artículo Open Access bajo la licencia CC BY-NC-ND (http:// creativecommons.org/licenses/by-nc-nd/4.0/).
\end{abstract}

\footnotetext{
* Autor para correspondencia.

Correo electrónico: pilivillamizar16@gmail.com (R. Villamizar-Durán).
} 


\section{KEYWORDS}

Pulmonary arterial

hypertension;

Ductus arteriosus;

Pregnancy

\section{Pulmonary hypertension of the newborn with in utero closure of the ductus arteriosus and twin reversed arterial perfusion sequence}

\begin{abstract}
Introduction: Pulmonary hypertension of the newborn secondary to in utero closure of the ductus arteriosus is a frequent complication in pregnant patients taking non-steroidal antiinflammatory drugs during pregnancy. We present a case of reversed arterial perfusion sequence in a twin pregnancy with evidence of survival of the acardiac twin with the blood flow of the pump twin. The treatment of choice is surgery with intrafetal laser coagulation on the acardiac twin, to prevent further complications on the healthy twin.

Clinical case: Twin with reversed arterial perfusion sequence. At 22 weeks gestation intrafetal laser coagulation on the acardiac twin was performed. The mother received acetaminophen for two days and non-steroidal anti-inflammatory drugs for the management of pain. Fetal echocardiogram was conducted at 31 weeks gestation, evidencing diastolic dysfunction of the right ventricle and ductus arteriosus closure. She underwent an emergency c-section; the newborn had pulmonary hypertension during the neonatal period that was solved with medical management.

Conclusion: Pulmonary hypertension of the newborn secondary to in utero closure of the ductus arteriosus due to drug consumption is an entity that poses a risk to the fetus, so a timely diagnosis and treatment is essential to improve survival of our patients.

(C) 2016 Sociedad Colombiana de Cardiología y Cirugía Cardiovascular. Published by Elsevier España, S.L.U. This is an open access article under the CC BY-NC-ND license (http:// creativecommons.org/licenses/by-nc-nd/4.0/).
\end{abstract}

\section{Introducción}

La hipertensión pulmonar del recién nacido es una entidad de múltiples etiologías ${ }^{1}$. Dentro de las causas posibles se conoce la muscularización excesiva de las arterias pulmonares, la cual a su vez puede ser secundaria a la hipoxia fetal, el estrés intrauterino crónico y la constricción intrauterina del ductus arterioso ${ }^{2}$, esto último dado usualmente por el uso de medicamentos tocolíticos del tipo de los analgésicos no esteroideos durante la gestación ${ }^{2,3}$, incluso ahora asociado también al uso del acetaminofén ${ }^{4}$.

En la etapa fetal la constricción del conducto arterioso es un proceso fisiopatológico anormal que lleva a la alteración de la estabilidad hemodinámica y la función cardiaca del feto. Estos fenómenos conducen a la hipertrofia del ventrículo derecho con posterior dilatación, falla cardiaca derecha, desarrollo de hidrops fetal, cierre total del conducto arterioso y muerte en el útero si no se llevan a cabo las intervenciones necesarias ${ }^{2}$.

La circulación con secuencia de perfusión arterial reversa en el embarazo gemelar twin reversed arterial perfusion, consiste en que el gemelo acárdico sobrevive gracias al flujo sanguíneo que el gemelo sano le aporta (gemelo bomba $)^{5}$. Se trata de una afección que ocurre en 1 de cada 35.000 partos, 1 de cada 100 embarazos gemelares monocigóticos y en 1 de cada 30 embarazos triples monocigóticos. Tres de cada cuatro casos de twin reversed arterial perfusion ocurren en gestaciones monocoriales biamnióticas ${ }^{5-7}$.

En la secuencia twin reversed arterial perfusion la sangre fluye desde la arteria umbilical del gemelo sano en dirección inversa hacia la arteria umbilical del gemelo acárdico a través de anastomosis arterioarteriales. El patrón de la circulación anormal lleva la sangre desoxigenada del gemelo bomba al gemelo acárdico a través de sus arterias ilíacas, las cuales llegan primero a la mitad inferior de su cuerpo y con dificultad a su porción superior, lo que contribuye a la presencia de las alteraciones estructurales características ${ }^{5}$.

Sin tratamiento, la mortalidad perinatal del gemelo bomba está entre el $50-75 \%$ debido al aumento de la demanda hemodinámica causada por el cortocircuito vascular, que conlleva a una falla cardiaca congestiva en el $28 \%$ de los $\operatorname{casos}^{6}$.

El cierre temprano del ductus arterioso incrementa la presión pulmonar antes del nacimiento, lo cual puede verse reflejado en la hipertensión pulmonar persistente del recién nacido con aumento de la morbimortalidad si no se diagnóstica a tiempo ${ }^{8}$.

Se presenta el caso de un recién nacido hijo de una madre de 28 años de edad, con embarazo gemelar, en quien se detectó una secuencia de perfusión arterial reversa gemelar con la presencia de un feto acárdico. A las 22 semanas, se le practicó fotocoagulación con láser de las anastomosis vasculares y vasos umbilicales del feto acárdico y por este motivo la madre recibió indometacina con el objetivo de prevenir un parto prematuro, al igual que dipirona y acetaminofén para manejo del dolor. Durante el seguimiento se detectaron los signos del cierre en el útero del ductus arterioso y la presencia de falla cardiaca derecha con pérdida del bienestar del feto sano por lo que a las 31 semanas la madre fue llevada a cesárea de emergencia.

La importancia de este artículo radica en el reconocimiento precoz de los signos del cierre del conducto arterioso en el útero que se puede presentar luego del uso de medicamentos antiinflamatorios tipo analgésicos no esteroideos para el manejo del dolor y el uso de la indometacina como tocolítico, incluso antes de la semana 32 de gestación, que 


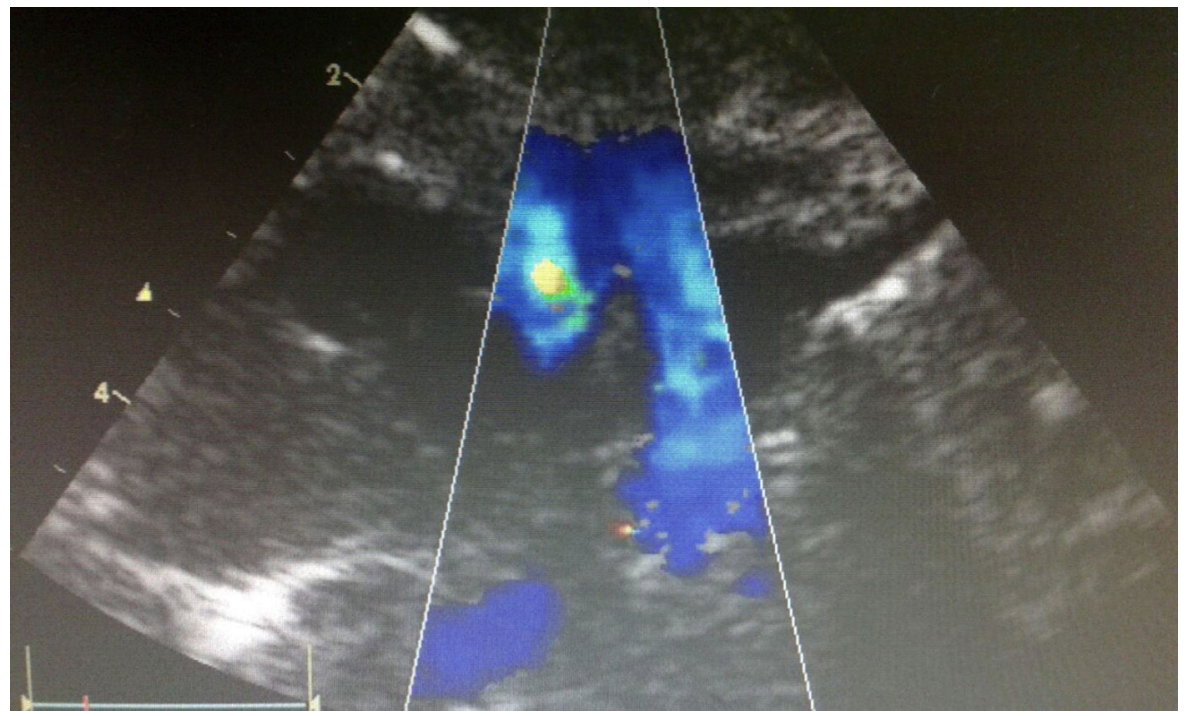

Figura 1 Ecocardiograma realizado al gemelo bomba a las 31 semanas de gestación con evidencia de ductus arterioso cerrado.

es la edad gestacional, de la cual la mayoría de los artículos comentan para el cierre precoz del ductus arterioso. Esta situación patológica cambia la dinámica adecuada del flujo sanguíneo en el corazón fetal provocando falla cardiaca derecha con alta posibilidad de muerte y pérdida fetal del gemelo bomba, en un embarazo de alto riesgo.

Este hallazgo demuestra la importancia del seguimiento estrecho que se hace con el ecocardiograma fetal y el momento más oportuno de la interrupción del embarazo, para hacer el manejo médico y salvar la vida del paciente.

\section{Caso clínico}

Gestante de 28 años de edad, con embarazo gemelar monocorial biamniótico con diagnóstico de secuencia twin reversed arterial perfusión desde las 12 semanas de gestación, feto número 1 vivo y feto número 2 acárdico y acefálico. A las 22 semanas se llevó la madre a fetoscopia para fotocoagulación con láser de anastomosis vasculares y vasos umbilicales del feto acárdico por signos de falla cardiaca en el feto normal. Durante la hospitalización la madre recibió indometacina $50 \mathrm{mg}$ cada 8 horas para inhibición uterina y prevención del parto prematuro, al igual que dipirona $1 \mathrm{~g}$ endovenosa cada 8 horas y acetaminofén $1 \mathrm{~g}$ cada 8 horas por vía oral para manejo del dolor. En seguimiento de alto riesgo obstétrico, se le practicó ecocardiograma fetal de seguimiento a las 31 semanas de gestación, donde se documentó la presencia de feto vivo con sospecha elevada de cierre del ductus arterioso, disfunción ventricular derecha severa y pérdida de bienestar fetal, motivo por el cual la madre fue llevada a la cesárea de urgencia. Se obtuvo un gemelo de sexo masculino, peso: $1.536 \mathrm{~g}$, talla: $39,5 \mathrm{~cm}$, perímetro cefálico: $28,5 \mathrm{~cm}$, APGAR: $8-9-10$, que evolucionó con dificultad respiratoria y requirió intubación orotraqueal con soporte ventilatorio. El recién nacido recibió una dosis de surfactante pulmonar, y se le realizó un ecocardiograma que evidenció la presencia de un ductus arterioso cerrado (fig. 1); así como disfunción ventricular derecha severa y signos indirectos de hipertensión pulmonar severa (figs. 2 y 3), con insuficiencia tricúspide severa y presión pulmonar calculada de $40 \mathrm{~mm} \mathrm{Hg}$ (de la insuficiencia tricúspide) más $8 \mathrm{~mm} \mathrm{Hg}$ de la aurícula derecha lo cual da $48 \mathrm{~mm} \mathrm{Hg}$ sobre $69 \mathrm{~mm}$ Hg de presión arterial sistólica (tensión arterial del paciente 69/39/51) lo que representa una presión pulmonar del $70 \%$ en relación a la presión sistémica.

Se inicia manejo en la unidad de cuidado intensivo neonatal con ventilación mecánica, milrinone y óxido nítrico con respuesta médica parcial por lo cual se adiciona manejo con sildenafil, con signos clínicos y ecocardiográficos hacia la mejoría.

A los 22 días de vida fue dado de alta de la unidad neonatal por evolución favorable con resolución de la falla cardiaca y de la hipertensión pulmonar. El gemelo número 2 acárdico, se observa con dos extremidades inferiores y tronco, aparente gastrosquisis y sexo indiferenciado (fig. 4).

\section{Discusión}

La circulación fetal con secuencia de la perfusión arterial reversa en el embarazo gemelar twin reversed arterial perfusion, consiste en la presencia de un gemelo acárdico que sobrevive gracias al flujo sanguíneo proveniente del otro gemelo sano, (gemelo bomba).

Se define como acárdica una malformación congénita fetal, proveniente de un embarazo monocigótico, cuya característica más relevante es la ausencia del corazón funcional en uno de los fetos. El desarrollo del feto acárdico se efectúa a expensas de la sangre bombeada por el otro gemelo y por esta razón, se describen como fetos parásitos. En estos embarazos, el cordón umbilical del feto acárdico sale directamente del cordón umbilical del gemelo sano, del cual obtiene su sangre, por lo cual se conoce al gemelo sin acárdica como gemelo bomba9 .

La hipertensión pulmonar persistente del recién nacido es un desorden caracterizado por la persistencia de la resistencia vascular pulmonar elevada en el período neonatal con: cortocircuito de derecha a izquierda a través del ductus arterioso y/o el foramen oval, disminución del flujo 


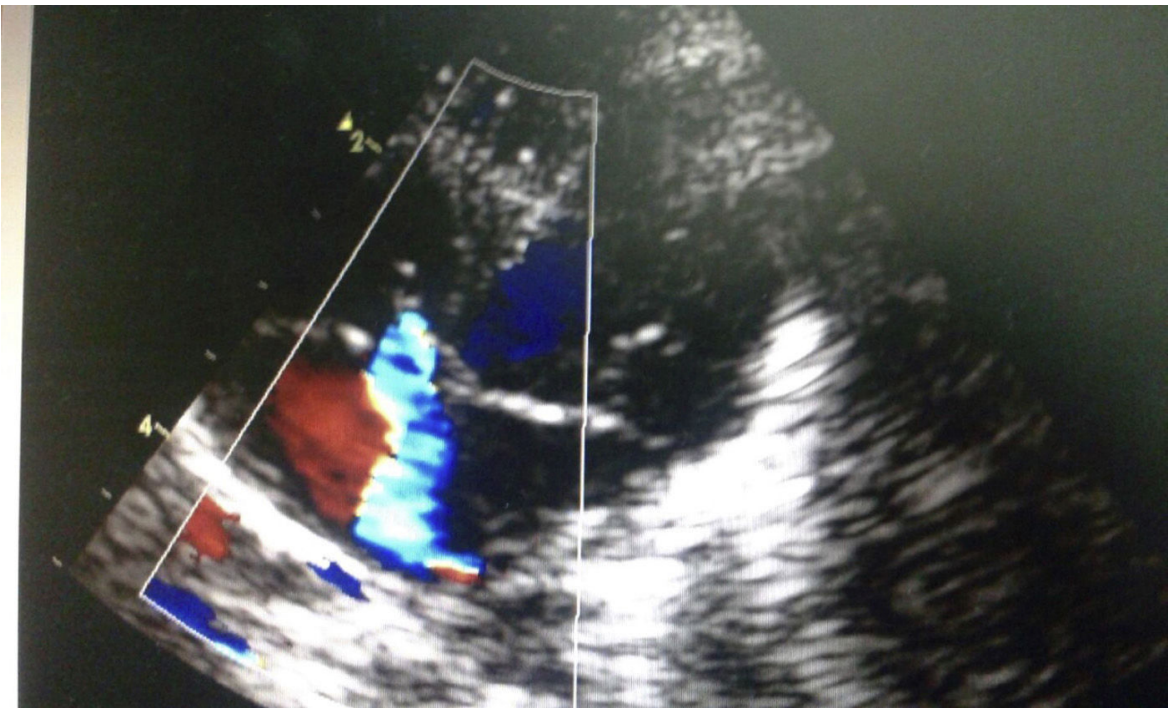

Figura 2 Ecocardiograma con evidencia de signos indirectos de hipertensión pulmonar dado por insuficiencia tricúspide severa.

pulmonar e hipoxemia sistémica, con antecedentes gestacionales o perinatales bien establecidos ${ }^{10}$.

Dentro de la etiología de la hipertensión pulmonar se postulan las siguientes: la muscularización excesiva de las arterias pulmonares como la que se presenta en la hipoxia fetal o el estrés intrauterino crónico; la constricción intrauterina del ductus arterioso secundaria a medicamentos como: aspirina, salicilatos, indometacina, ibuprofeno entre otros antiinflamatorios no esteroideos, así como los medicamentos tipo paracetamol (especialmente en el último trimestre de la gestación), que se han encontrado recientemente asociados al cierre prematuro del ductus arterioso ${ }^{10}$.

El ductus arterioso es una estructura vascular que conecta la aorta descendente proximal con la rama izquierda de la arteria pulmonar ${ }^{1}$. Durante la vida intrauterina el ventrículo derecho fetal eyecta $60-65 \%$ del gasto cardiaco combinado, del cual el $90 \%$ es desviado por el ductus arterioso hacia la aorta, garantizando con ello el flujo sistémico al feto en desarrollo ${ }^{11}$.

El ductus arterioso permanece abierto debido a las tensiones bajas del oxígeno y los niveles circulantes de la prostaglandina E2 y PGI2 producidas por la placenta ${ }^{3}$. Al nacimiento, caen las resistencias pulmonares y se incrementa la resistencia vascular periférica, aumentando el

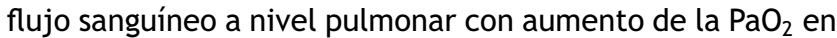
el endotelio ductal que activa el citocromo $\mathrm{P}_{450}$ con la subsecuente síntesis de la endotelina 1 que inicia la constricción del mismo ${ }^{12}$.

Si el ductus arterioso se cierra en el período intrauterino, puede aparecer la hipertensión pulmonar grave con la insuficiencia cardiaca congestiva e incluso la muerte fetal. Actualmente, no se conoce ninguna intervención intrauterina para permeabilizar de nuevo un ductus arterioso fetal cerrado $^{2}$.



Figura 3 Ecocardiograma con imagen doppler que evidencia presión pulmonar calculada en $40 \mathrm{mmHg}$ con presión sistémica 69/39/51 lo que representa 70\% de la presión pulmonar con relación a la presión sistémica. 


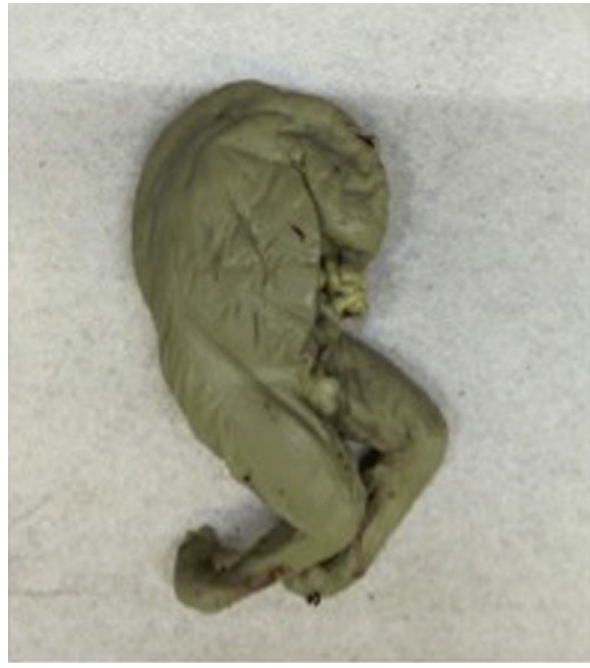

Figura 4 Gemelo acárdico tras inclusión en formol.

El flujo normal a través del ductus arterioso debe ser homogéneo y uniforme, y la velocidad en el útero no debe ser mayor a los $1,4 \mathrm{~m} / \mathrm{s}$. Se considera que existe un flujo restrictivo cuando las velocidades de flujo sistólico y diastólico están elevadas para la edad gestacional ${ }^{3}$. Cuando el flujo a través del conducto arterioso es restrictivo, se produce por un aumento de las presiones de las cavidades cardiacas derechas y posteriormente la regurgitación tricúspide que puede ser de leve a moderada, con la insuficiencia pulmonar de moderada a severa y la dilatación e hipertrofia del ventrículo derecho con aumento de la presión de la aurícula derecha, llevando a la falla ventricular derecha, dado que el corazón fetal no está preparado para enfrentarse a una alteración hemodinámica que se instaura de forma súbita ${ }^{10}$.

Durante el embarazo comúnmente se usan los medicamentos antiinflamatorios no esteroideos como: la indometacina, el diclofenaco, el ibuprofeno, el naproxeno, la nimesulida y la aspirina. Este tipo de medicamentos actúa bloqueando la síntesis de las prostaglandinas E2, F2, alfa, $12^{4}$.

La indometacina en especial, tiene un profundo efecto en la función de las plaquetas y de los neutrófilos, así como en la hemodinámica renal, gastrointestinal, cerebral y mesentérica ${ }^{13}$. En el feto se ha reportado el cierre prematuro del ductus con el uso de la indometacina, especialmente, luego del segundo trimestre cuando el medicamento atraviesa la placenta y se encuentran en el feto niveles sanguíneos de la indometacina similares a los de la madre ${ }^{13,14}$. Dentro de las complicaciones del uso de la indometacina también se ha documentado: la disminución del gasto urinario con oligohidramnios, la enterocolitis necrozante, la perforación ileal, la hemorragia intracraneana y la hipertensión pulmonar. Sin embargo, estas complicaciones dependen del tiempo de exposición a la indometacina y de la edad gestacional del feto en el momento de uso del medicamento. Se ha encontrado que en fetos mayores de 31 semanas la posibilidad del cierre prematuro del ductus y la hipertensión pulmonar se presenta más comúnmente que en pacientes con edad gestacional menor a las 30 semanas (específicamente la tasa de respuesta es del $50 \%$ en los pacientes de 32 semanas de edad gestacional y hasta el $100 \%$ en los mayores de 34 semanas de edad gestacional); la suspensión del uso del medicamento tiende a revertir el oligohidramnios y la constricción del ductus (en el caso de que el ductus no se haya cerrado por completo) ${ }^{13-15}$.

Paradójicamente también se ha encontrado que la exposición prenatal a la indometacina puede ocasionar la constricción parcial del ductus arterioso provocando una daño en la pared que resulta en la patencia del mismo luego del nacimiento, que requiere el cierre quirúrgico del ductus arterioso persistente dado que no hay respuesta clínica con el manejo farmacológico en la etapa neonatal ${ }^{13}$.

También se demostró en modelos animales que el consumo materno del té casero (Camelliasinensis), así como el té negro, el té verde, el té de boldo y la yerba mate, causan constricción ductal por mecanismos similares a los que intervienen en la síntesis de las prostaglandinas ${ }^{4}$, por lo que estas bebidas deben ser evitadas durante la gestación.

En este artículo se presenta el caso de una gestación gemelar con gemelo acárdico con secuencia de perfusión reversa que al comportarse como un síndrome de transfusión feto a feto, cursa en el donante con hipoxia lo cual lo hace más sensible al uso de la indometacina y al cierre en el útero del ductus arterioso, la elevación de la postcarga del ventrículo derecho, el aumento de la presión de fin de diástole de dicho ventrículo, con insuficiencia tricúspidea severa (en este caso presión pulmonar calculada del $70 \%$ de la presión sistémica), aumento de la presión de la aurícula derecha, así como aumento del flujo del tronco de la pulmonar e hipertrofia de la capa muscular a nivel de la vasculatura pulmonar, todo lo cual conduce a la hipertensión pulmonar $^{12,15}$.

Por lo anterior, las gestaciones gemelares deben ser monitorizadas estrechamente con el ecocardiograma fetal desde la semana 20 o si hay el antecedente del uso de medicamentos tocolíticos como la indometacina, especialmente, cuando esta se usa luego de las 32 semanas de gestación o cerca al día del parto, dado las múltiples comorbilidades ya mencionadas ${ }^{3,13}$.

El manejo de la persistencia de la circulación fetal después del nacimiento y la hipertensión pulmonar exigen hospitalizaciones prolongadas en la unidad de cuidado intensivo neonatal, pero tienen una buena respuesta clínica si se diagnóstica el cierre prematuro del ductus arterioso de manera oportuna y se maneja inmediatamente cuando hay evidencia de la falla cardiaca o signos de polihidramnios ${ }^{3,13}$.

\section{Responsabilidades éticas}

Protección de personas y animales. Los autores declaran que para esta investigación no se han realizado experimentos en seres humanos ni en animales.

Confidencialidad de los datos. Los autores declaran que han seguido los protocolos de su centro de trabajo sobre la publicación de datos de pacientes.

Derecho a la privacidad y consentimiento informado. Los autores han obtenido el consentimiento informado de los pacientes y/o sujetos referidos en el artículo. Este documento obra en poder del autor de correspondencia. 


\section{Conflicto de intereses}

\section{Los autores declaran no tener ningún conflicto de intereses.}

\section{Bibliografía}

1. Gasque JJ. Hipertensión pulmonar persistente del recién nacido. Revista Mexicana de Pediatría. 2009;76:220-30.

2. Aslam M, Christou HA. Intrauterine Closure of the Ductus Arteriosus: Implications for the Neonatologist. American Journal of Perinatology. 2009;26:7.

3. Luchesi S, Manica JL, Zielinsky P. Intrauterine ductus arteriosus constriction. Analysis of a historic cohort of 20 cases. Arq Bras Cardiol. 2003;81:405-10.

4. Araújo J. Conducto arterioso restrictivo tras el consumo de acetaminofén. Rev Colomb Cardiol. 2014;21: 350-5.

5. Holland MG, Mastrobattista JM, Lucas MJ. Diagnosis and management of twin reversed arterial perfusion sequence. Up to date. 2013.

6. Simpson LL. Ultrasound in twins: dichorionic and monochorionic. Semin Perinatol. 2013;37:348-58.

7. Corbacioglu A, Gul A, Bakirci IT, Gedikbasi A, Yildirim G. Treatment of twin reversed arterial perfusion sequence with alcohol ablation or bipolar cord coagulation. Int J Gynaecol Obstet. 2012;117:257-9.

8. Takahashi H, Takahashi S, Tsukamoto K, Ito Y, Nakamura T, Hayashi $\mathrm{S}$, et al. Persistent pulmonary hypertension of the newborn in twin-twin transfusion syndrome following fetoscopic laser surgery. The Journal of Maternal-Fetal and Neonatal Medicine. 2012;25:543-5.

9. Ramírez L, Carmona de Uzcategui ML, Chacin B, Moreno F. Síndrome de perfusión arterial retrógrada en gemelos: feto arcádico. Reporte de un caso. Rev Obstet Ginecol Venez. 2009;69:193-203.

10. Van Marter L, Hernández-Díaz S, Werler M, Louik C, Mitchell A. Nonsteroidal Antiinflammatory Drugs in Late Pregnancy and Persitent Pulmonary Hypertension of the Newborn. Pediatrics. 2013:131.

11. Gewilling M, Brown S, De Catte L, Debeer A, Eyskens B, Cossey $\mathrm{V}$, et al. Premature fetal closure of the arterial duct: clinical presentations and outcome. European Heart Journal. 2009;30:1530-6.

12. Shehata B, Bare J, Denton T, Habib M, Black J. Premature closure of the ductus arteriosus: variable response among monozygotic twin safter in utero exposure to indomethacin. Fetal and Pediatric Pathology. 2006;25:151-7.

13. Norton ME, Teratogen Update. Fetal Effects of Indomethacin Administration During Pregnancy. Teratology. 1997;56: 282-92.

14. Silvani P, Camporesi A. Drug Induced Pulmonary Hypertension in Newborns: A Review. Current Vascular Pharmacology. 2007;5:129-33.

15. Mushiake K, Motoyoshi F, Kinoshita Y, Nakagawa A, Ito M. Severe heart failure due to ductal constriction caused by maternal indomethacin. Pediatrics International. 2002;44:174-6. Primera pág. 